\title{
Improvement in Hyperglycemia Prevents Surgical Site Infection Irrespective of Insulin Therapy in Non-diabetic Patients Undergoing Gastrointestinal Surgery
}

\author{
Ayami Yoneda $^{1} \cdot$ Yoshio Takesue $^{2}$ (1) Yoshiko Takahashi $^{1} \cdot$ Kaoru Ichiki $^{2} \cdot$ \\ Toshie Tsuchida $^{2} \cdot$ Hiroki Ikeuchi $^{3} \cdot$ Motoi Uchino $^{3} \cdot$ Etsuro Hatano $^{4} \cdot$ Hisashi Shinohara $^{4}$. \\ Naohiro Tomita ${ }^{4}$
}

Published online: 23 January 2020

(C) The Author(s) 2020

\begin{abstract}
Background Intensive glycemic control is recommended to prevent surgical site infections (SSI). Our aim was to evaluate retrospectively the effect of improvement in hyperglycemia irrespective of insulin use on the incidence of SSI in non-diabetic patients.

Methods The highest blood glucose (BG) concentration within $12 \mathrm{~h}$ (early peak BG) and the final BG from 12 to $24 \mathrm{~h}$ after surgery were evaluated in patients who underwent gastrointestinal surgery. Patients with an early peak BG of $\geq 150 \mathrm{mg} / \mathrm{dL}$ were divided into those with persistent (final BG of $\geq 150 \mathrm{mg} / \mathrm{dL}$ ) and improved hyperglycemia (final $\mathrm{BG}$ of $<150 \mathrm{mg} / \mathrm{dL}$ ). Patients without hyperglycemia and those with late-onset hyperglycemia were also assessed for SSI risk.

Results Overall, 1612 patients were studied (diabetes, $n=293$ ). Although hyperglycemia increased the SSI rates in non-diabetic patients, no correlation was demonstrated in patients with diabetes at any cutoff final BG defining htperglycemia except for $180 \mathrm{mg} / \mathrm{dL}$. Hyperglycemia improved without insulin therapy in 283 of 512 non-diabetic patients who had early hyperglycemia. The adjusted standardized residual for those with SSI and persistent hyperglycemia was $5.2(P<0.05)$. In contrast, the absence of hyperglycemia was a significant preventive factor for SSI. In the multivariate analyses, persistent hyperglycemia was an independent risk factor for SSI (odds ratio 1.54; $95 \%$ confidence interval 1.03-2.31).

Conclusions Remission of hyperglycemia within $24 \mathrm{~h}$ after surgery prevented SSI in non-diabetic patients. Considering that hyperglycemia improved in approximately half of patients without insulin therapy, commencement of insulin dosing after two consecutive BGs of $\geq 150 \mathrm{mg} / \mathrm{dL}$ might be reasonable, especially in general wards.
\end{abstract}

Yoshio Takesue

takesuey@hyo-med.ac.jp

1 Department of Pharmacy, The Hospital of Hyogo College of Medicine, Nishinomiya, Hyogo, Japan

2 Department of Infection Control and Prevention, Hyogo College of Medicine, 1-1 Mukogawa-cho, Nishinomiya, Hyogo 663-8501, Japan

3 Department of Inflammatory Bowel Disease, Hyogo College of Medicine, Nishinomiya, Hyogo, Japan

4 Department of Surgery, Hyogo College of Medicine, Nishinomiya, Hyogo, Japan

\section{Introduction}

Several observational studies have shown an association between hyperglycemia and postoperative complications including surgical site infection (SSI) [1-5]. Acute hyperglycemia can significantly alter the innate immune responses to infection in surgical patients, resulting in SSI [6]. Some studies evaluated the highest blood glucose concentration (BG) after surgery as a cause of SSI [5]. However, SSI can be prevented by controlling the BG with insulin [1, 3, 7], and transient hyperglycemia immediately after completion of surgery may not cause SSI. A meta- 
analysis revealed that target BGs of both $<110$ and $110-150 \mathrm{mg} / \mathrm{dL}$ showed a significant benefit in reducing SSI for these intensive insulin protocols compared with a conventional protocol [1]. The guidelines established by the American College of Surgeons and Surgical Infection Society [8] recommended that the target $\mathrm{BG}$ should range from 110 to $150 \mathrm{mg} / \mathrm{dL}$. However, there is compelling agreement that stress hyperglycemia should be treated in all surgical patients [9].

Stress hyperglycemia arises as a result of a cascade of normal physiologic responses to a surgical insult [6], and the effect of surgical stress can be attenuated over time, leading to achievement of the target BG the next morning in some non-diabetic patients who are not undergoing insulin therapy. Considering the significantly higher risk of hypoglycemia in association with an intensive insulin protocol, the target BG the morning after surgery might not be equal to the BG needed to start insulin therapy, especially in a non-surgical intensive care unit (ICU) setting. The present study was performed to investigate the effect of improvement in hyperglycemia irrespective of insulin therapy on SSI and assess the natural remission of hyperglycemia within $24 \mathrm{~h}$ after surgery in non-diabetic patients.

\section{Methods}

This retrospective study was conducted in three surgical wards from January 2014 to January 2016 and was approved by the Institutional Review Board of Hyogo College of Medicine (No. 2284). The inclusion criteria were age 15 years or older, performance of gastroenterological surgery, survival for at least 5 days, and measurement of BG at least twice. Patients receiving steroids were excluded because steroids can increase BG concentrations. The highest BG within $12 \mathrm{~h}$ (early peak BG) and the final $\mathrm{BG}$ from 12 to $24 \mathrm{~h}$ after surgery were evaluated. In accordance with hospital protocols, laboratory data, including BG, were measured at least when patients were admitted to the ward after surgery and the day after surgery. No BG measurements were taken with point-of-care testing of capillary blood; all BG data were measured using laboratory analyzers or arterial blood gas analyzers. Patients with a history of diabetes or hemoglobin A1c $(\mathrm{HbA} 1 \mathrm{c})>6.5 \%$ were classified as having diabetes.

Hyperglycemia was defined by several cutoff BGs (110, $150,180$, and $200 \mathrm{mg} / \mathrm{dL})$. SSI rates were evaluated in patients with and without diabetes according to final BG categories $(<12.5$ th percentile, 12.5 th -25 th percentile, 25 th-37.5th percentile, 37.5 th-50th percentile, 50th-62.5th percentile, 62.5 th-75th percentile, 75 th-87.5th percentile, and $\geq 87.5$ th percentile). For evaluation of the effect of $B G$ control, patients with an early peak $B G$ of $\geq 150 \mathrm{mg} / \mathrm{dL}$ were divided into those with persistent hyperglycemia (final $\mathrm{BG}$ of $\geq 150 \mathrm{mg} / \mathrm{dL}$ ) and improved hyperglycemia (final $\mathrm{BG}$ of $<150 \mathrm{mg} / \mathrm{dL}$ ). Patients without hyperglycemia throughout the postoperative period (within $24 \mathrm{~h}$ postoperatively) (early peak and final $\mathrm{BG}$ of $<150 \mathrm{mg} / \mathrm{dL}$ ) and those with late-onset hyperglycemia (early peak BG of $<150 \mathrm{mg} / \mathrm{dL}$, final $\mathrm{BG}$ of $\geq 150 \mathrm{mg} / \mathrm{dL}$ ) were also assessed for SSI risk. Commencement of insulin dosing according to peak BG categories $(<110,110-149$, 150-179, 180-199 mg/dL, and $\geq 200 \mathrm{mg} / \mathrm{dL}$ ) was evaluated. Insulin was used at the clinician's discretion, and no interventions for BG control were mandated.

SSI was diagnosed based on definitions stated in the guidelines issued by the National Nosocomial Infections Surveillance System [10]. The wounds were inspected daily by a nurse, once a week by a surveillance member, once during the hospital stay by an attending physician, and at the 4-week postoperative follow-up visits. The criteria for the diagnosis of SSI included an infection that occurred within 30 days after the operation and at least one of the following: (1) purulent discharge from the incision or from a drain placed into the organ/space; (2) organisms could be isolated by culturing fluid or tissue from the incision or the organ/space; (3) an open wound with signs and symptoms of infection; and (4) an abscess or other evidence of infection found on examination of the incision or the organ/space. In this series, the 75 th percentile was used for the definition of prolonged surgery in each procedure, massive intraoperative hemorrhage, and long preoperative hospital stay.

Adjusted standardized residuals for performing post hoc tests were used in the comparison of SSI rate among the four BG control groups. Absolute values greater than the critical value of 1.96 have raw $P$ values of less than 0.05 . Univariate analyses for risk factors associated with SSI were performed by the Chi-square test, and potential confounders were examined by cross-tabulation. Variables selected by these univariate analyses $(P<0.1)$ were subsequently entered into a logistic regression model to estimate the size of the association [odds ratio (OR)] and the 95\% confidence interval (CI). SPSS v.21.0 for Windows (IBM Corp., Armonk, NY, USA) was used for all analyses, and the level of significance was set at $P<0.05$.

\section{Results}

Overall, 1899 patients underwent gastrointestinal surgery, of whom 1612 (male, $n=1043$, diabetes, $n=293$ ) were included in the analyses. Patients who did not survive at least 5 days after surgery (one patient), did not have the required two measurements of BG (127 patients) or were receiving steroids (159 patients) were excluded. The mean 
age was $59.6 \pm 17.8$ years. Patients with an American Society of Anesthesiologists physical status score of $\geq 3$ comprised $21.3 \%$ of the total patients. Preoperative hemoglobin A1c levels of the patients with diabetes were as follows: 61 with $<6.5 \%, 111$ with $6.5-6.9 \%, 81$ with $7.0-7.9 \%$, and 40 with $\geq 8.0 \%$. In total, 136 patients were admitted to the ICU after surgery. Colorectal surgery was the most frequently performed surgery $(n=572)$, followed by small intestinal surgery $(n=324)$, hepatobiliary pancreatic surgery $(n=329)$, gastric surgery $(n=279)$, esophageal surgery $(n=49)$, and other surgeries $(n=59)$. The 28-day mortality rate was $0.37 \%$.

SSI occurred in 231 patients (14.3\%), 67 of whom had incisional SSI, whereas 164 had organ/space SSI. The SSI rate was $14.0 \%$ in non-diabetic patients and $16.0 \%$ in patients with diabetes. Median length of hospital stay was 20 days, and there was a significant difference between patients with and without SSI (41 days versus 18 days, $P<0.001$ ).

The incidence of hyperglycemia as assessed by the early peak BG in non-diabetic patients was significantly lower than that in patients with diabetes at each cutoff value $(110 \mathrm{mg} / \mathrm{dL}: 79.4 \%$ vs. $94.9 \% ; 150 \mathrm{mg} / \mathrm{dL}: 38.8 \%$ vs. $74.1 \% ; 180 \mathrm{mg} / \mathrm{dL}: 16.1 \%$ vs. $52.2 \% ; 200 \mathrm{mg} / \mathrm{dL}: 7.0 \%$ vs $35.2 \% ; P<0.001$ each). The number of patients with diabetes who were treated by insulin according to the peak BG categories was $5 / 15(33.3 \%)$ for $<110 \mathrm{mg} / \mathrm{dL}, 21 / 61$ $(34.4 \%)$ for $110-149 \mathrm{mg} / \mathrm{dL}, \quad 32 / 64 \quad(50.0 \%)$ for $150-179 \mathrm{mg} / \mathrm{dL}, 30 / 50(60.0 \%)$ for $180-199 \mathrm{mg} / \mathrm{dL}$, and $86 / 103(83.5 \%)$ for $\geq 200 \mathrm{mg} / \mathrm{dL}$. However, a low performance rate was observed in non-diabetic patients $[0 / 272$ (0.0\%), 1/535 (0.2\%), 3/300 (0.1\%), 4/120 (3.3\%), and $8 / 92(8.7 \%)$, respectively].

There were 679 non-diabetic patients and 37 diabetic patients who never developed hyperglycemia, 287 nondiabetic patients and 55 diabetic patients with early hyperglycemia with resolution, 225 non-diabetic patients and 162 diabetic patients with persistent hyperglycemia, and 128 non-diabetic patients and 39 diabetic patients with late-onset hyperglycemia. Among 512 non-diabetic patients with an early peak BG of $\geq 150 \mathrm{mg} / \mathrm{dL}$, hyperglycemia improved in $55.3 \%$ of patients without insulin therapy. BG significantly decreased from $177.6 \pm 30.1$ to $127.5 \pm 14.4 \mathrm{mg} / \mathrm{dL}$ without insulin use in patients with improved hyperglycemia $(P<0.001)$. The SSI rate was $24.9 \%$ in non-diabetic patients with persistent hyperglycemia, $14.3 \%$ in those with improved hyperglycemia, $14.1 \%$ in those with late-onset hyperglycemia, and $10.2 \%$ in those without hyperglycemia (Fig. 1). The adjusted standardized residual for those with SSI and persistent hyperglycemia was $5.2(P<0.05)$. In contrast, the adjusted standardized residual for those with SSI and without hyperglycemia was -4.1 ; thus, the absence of hyperglycemia was a significant preventive factor for SSI $(P<0.05)$. In diabetic patients, persistent hyperglycemia also increased SSI rate compared with other BG control groups (the adjusted standardized residual, 2.6) (Fig. 1).

A significant difference was found between the early peak BG and final BG $(150.7 \pm 46.2$ vs. $144.2 \pm 40.6 \mathrm{mg} /$ $\mathrm{dL}$, respectively, $P<0.001)$. The SSI rate was compared between patients with and without hyperglycemia according to the different cutoff early peak BG within $12 \mathrm{~h}$ and final BG (Fig. 2). Below and above cutoff final BG values discriminated the SSI rate between patients better than did below and above cutoff early peak BG values. When a cutoff level of $200 \mathrm{mg} / \mathrm{dL}$ was adopted, the SSI rate was not significantly different between patients with below and above cutoff BG values for the early peak BG (17.4\% vs. $13.9 \%$, respectively, $P=0.187$ ); however, a significantly higher SSI rate was demonstrated for the final BG $(22.3 \%$ vs. $13.5 \%, P=0.004)$.

In non-diabetic patients, the SSI rates increased incrementally with higher final BG categories $(<12.5$ th percentile, $8.0 \%$; 12.5 th-25th percentile, $9.0 \% ; 25$ th- 37.5 th percentile, $11.3 \%$; 37.5th-50th percentile, $11.0 \%$; 50th62.5th percentile, $14.8 \%$; 62.5th-75th percentile, $14.7 \%$; 75th-87.5th percentile, $20.5 \%$; $\geq 87.5$ th percentile, $21.8 \%$ ). However, patients with diabetes did not show an increase in the SSI rate in a dose-response manner as the BG categories increased (Fig. 3). Hyperglycemia assessed by final BG was associated with higher SSI rates in non-diabetic patients. However, no correlation between hyperglycemia and the SSI rate was found in patients with diabetes at any cutoff final BG values defining hyperglycemia except for $180 \mathrm{mg} / \mathrm{dL}$ (Fig. 4). Hence, further evaluation of the effect of BG control on the SSI rate was conducted only in nondiabetic patients in this study.

Table 1 shows the results of univariate analyses for the risk factors associated with SSI in non-diabetic patients. Common risk factors including American Society of Anesthesiologist physical status classification $\geq 3$ (SSI rate, $18.5 \%$ ), prolonged surgery (SSI rate, 23.3\%), and wound class $\geq 3$ (SSI rate, 22.8\%) were identified. Persistent hyperglycemia and a final $\mathrm{BG}$ of $\geq 150 \mathrm{mg} / \mathrm{dL}$ were associated with an increased risk of SSI, whereas the absence of hyperglycemia throughout the postoperative period was associated with a decreased risk of SSI. In the multivariate analyses, independent risk factors for SSI were persistent hyperglycemia (OR 1.54; 95\% CI 1.03-2.31), prolonged surgery, blood transfusion, preoperative infections, hepatectomy with biliary tract resection and other types of hepatobiliary pancreatic surgery, and esophageal surgery. Small intestinal surgery and laparoscopic surgery were associated with a lower risk (Table 2).

The various gastrointestinal surgical interventions carried out in the study cohort were found to carry varying 


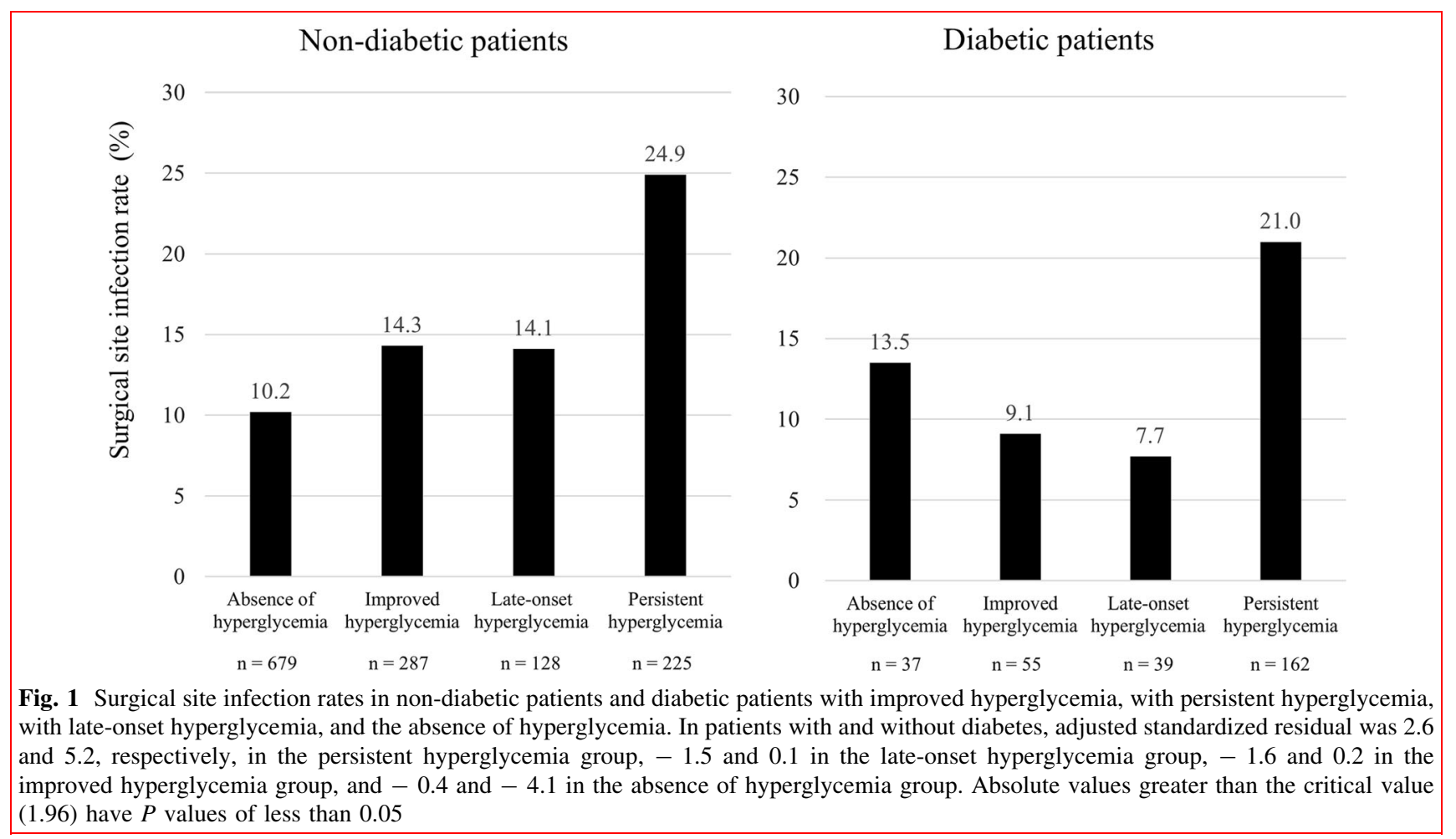

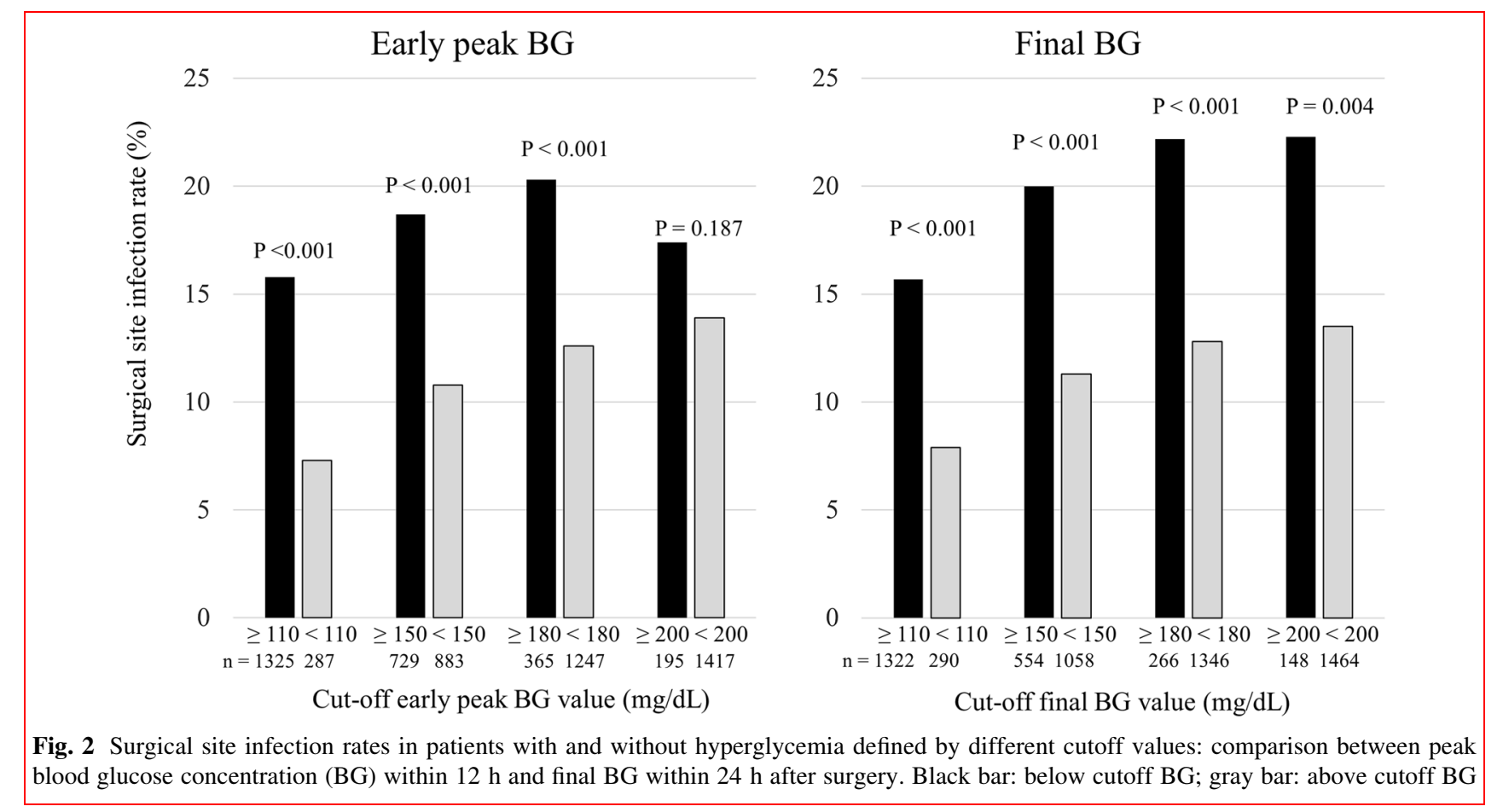

risks of SSI (13.2\% for colorectal surgery; $6.9 \%$ for small intestine, $15.4 \%$ for gastric surgery; $32.4 \%$ for esophageal surgery; $21.8 \%$ for hepatobiliary pancreatic surgery; and $9.6 \%$ for other surgery). Therefore, the risks of SSI according to category of BG control were further investigated for each surgical procedure (Table 3). Persistent hyperglycemia (OR 1.88, 95\% CI 0.94-3.78, $P=0.072$ ) in patients undergoing colorectal surgery, the absence of 


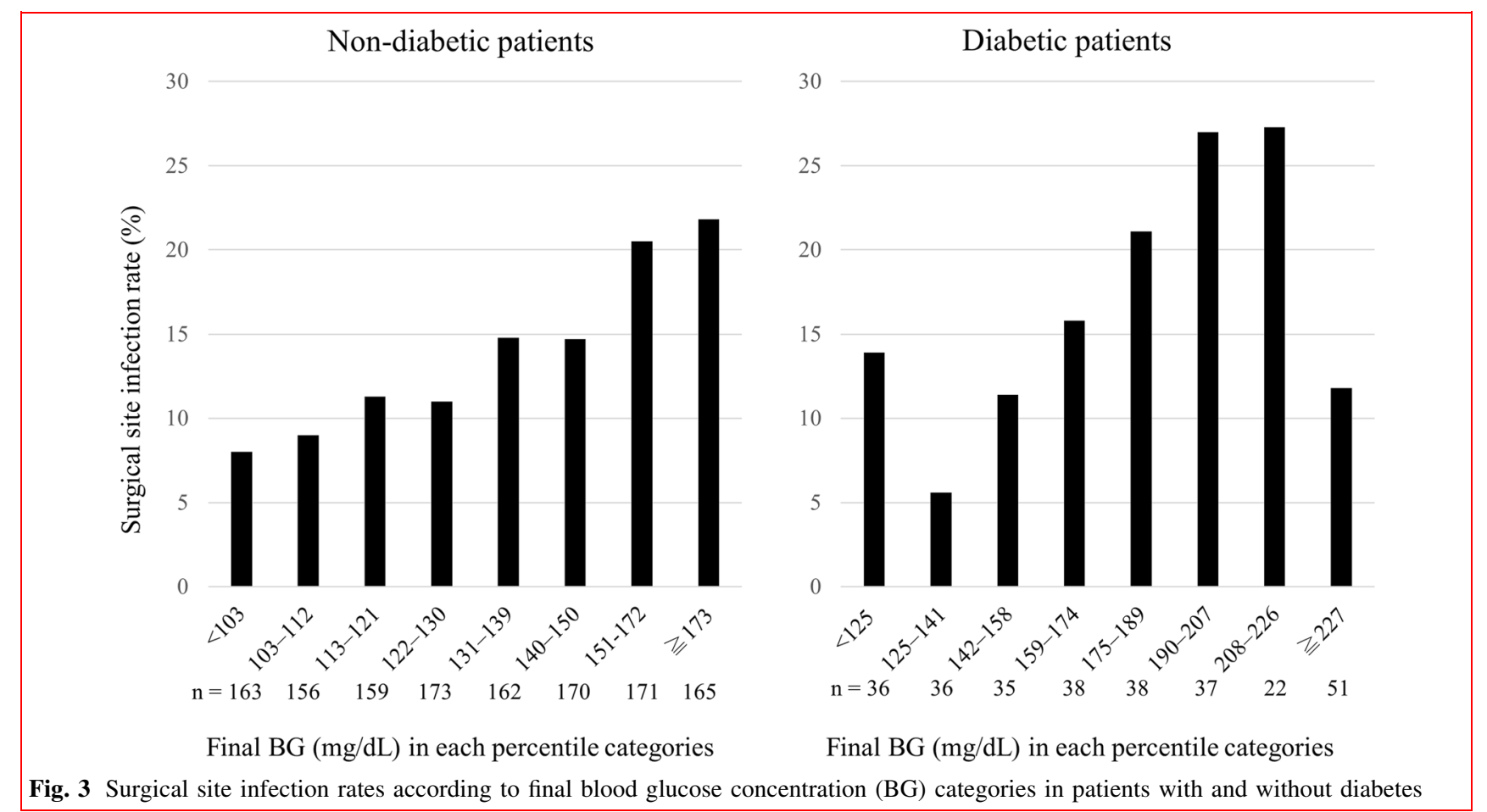

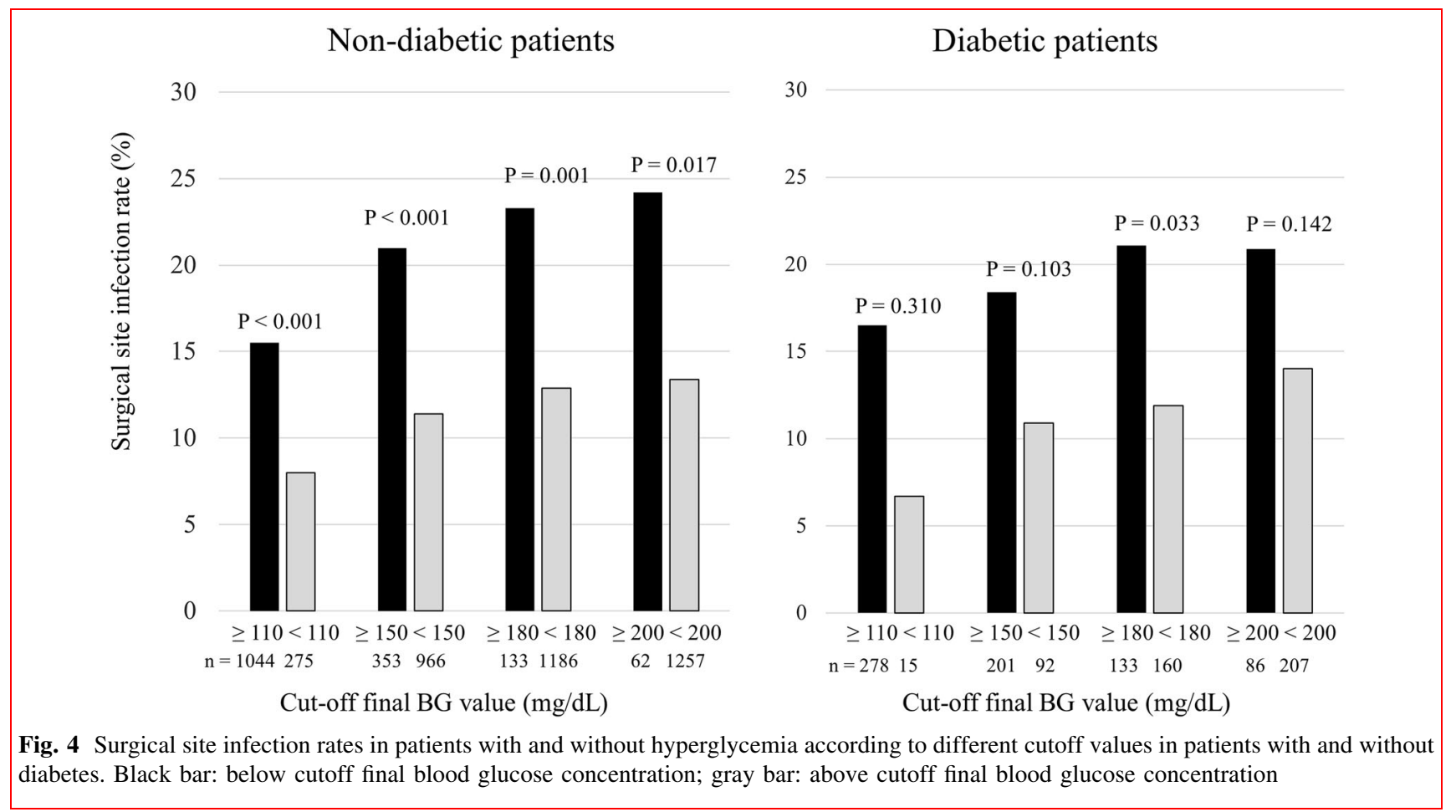

hyperglycemia (OR $0.39,95 \%$ CI $0.15-0.99, P=0.042$ ) and late-onset hyperglycemia (OR 2.96, 95\% CI 1.01-8.72, $P=0.040$ ) in patients undergoing small intestine surgery, and persistent hyperglycemia (OR 4.14, 95\% CI 1.94-8.83,
$P<0.001$ ), late-onset hyperglycemia (OR $0.25,95 \%$ CI 0.06-1.07, $P=0.052$ ), and improved hyperglycemia (OR $0.18,95 \%$ CI $0.04-0.78, P=0.011$ ) for those undergoing gastric surgery were selected by univariate analysis for 
Table 1 Risk factors associated with surgical site infection in non-diabetic patients: univariate analyses

\begin{tabular}{lll}
\hline Factors & SSI, no of patients (\%) & $P$ value \\
\cline { 2 - 3 } & Yes & No \\
\hline
\end{tabular}

(a) Demographics and comorbidity

Male

Age $\geq 65$

Hypertension

Chronic renal dysfunction

Chronic hepatic dysfunction

Cardiac disease

ASA physical status classification $\geq 3$

(b) Preoperative factors

Smoking

Prolonged preoperative hospital stay

Immunosuppressant

Biological products

Anti-cancer drug

Preoperative serum albumin $<3.0 \mathrm{~g} / \mathrm{dL}$

Preoperative anemia

Body mass index $\geq 25$

Preoperative infection

Preoperative $\mathrm{BG} \geq 150 \mathrm{mg} / \mathrm{dL}$

(c) Primary surgery

Colorectal surgery

Construction or closure of stoma

Surgery for small intestine

Gastric surgery

Esophageal surgery

Hepatectomy without biliary tract resection

Hepatectomy combined with biliary tract resection

Surgery for pancreas

Cholecystectomy

Other hepatobiliary pancreatic surgery

Other surgery

(d) Intra- and postoperative factors

Wound class $\geq 3$

Prolonged surgery

Emergent surgery

Laparoscopic surgery

Massive intraoperative bleeding

Perioperative blood transfusion

Intraoperative hypothermia $\left(<36{ }^{\circ} \mathrm{C}\right)$

Admission to intensive care unit

Insulin treatment

(e) Classification of blood glucose concentration control

Absence of hyperglycemia

Improved hyperglycemia

Persistent hyperglycemia

Late-onset hyperglycemia

\begin{tabular}{|c|}
\hline $119 / 829(14.4)$ \\
\hline 98/558 (17.6) \\
\hline $53 / 304(17.4)$ \\
\hline $9 / 34(26.5)$ \\
\hline $30 / 175(17.1)$ \\
\hline 21/121 (17.4) \\
\hline $42 / 227(18.5)$ \\
\hline $71 / 476(14.9)$ \\
\hline $51 / 288(17.7)$ \\
\hline $32 / 254(12.6)$ \\
\hline $0 / 15(0.0)$ \\
\hline $8 / 30(26.7)$ \\
\hline $32 / 151(21.2)$ \\
\hline $101 / 516$ (19.6) \\
\hline $23 / 176(13.1)$ \\
\hline 43/89 (48.3) \\
\hline 7/41 (17.1) \\
\hline $64 / 485(13.2)$ \\
\hline $41 / 264(15.5)$ \\
\hline 21/304 (6.9) \\
\hline $34 / 221(15.4)$ \\
\hline $12 / 37(32.4)$ \\
\hline $12 / 87(13.8)$ \\
\hline $16 / 36(44.4)$ \\
\hline $11 / 33(33.3)$ \\
\hline $1 / 45(2.2)$ \\
\hline $8 / 19(42.1)$ \\
\hline $5 / 52(9.6)$ \\
\hline $23 / 101(22.8)$ \\
\hline $63 / 270(23.3)$ \\
\hline $19 / 133(14.3)$ \\
\hline $11 / 185(5.9)$ \\
\hline $80 / 304(26.3)$ \\
\hline $59 / 167(35.3)$ \\
\hline $36 / 243(14.8)$ \\
\hline $34 / 136(25.0)$ \\
\hline $4 / 16(25.0 \%)$ \\
\hline $69 / 679(10.2)$ \\
\hline $41 / 287(14.3)$ \\
\hline $56 / 225(24.9)$ \\
\hline $18 / 128(14.0)$ \\
\hline
\end{tabular}

$65 / 490(13.3)$

0.581

$86 / 761(11.3)$

$131 / 1015$ (12.9)

$175 / 1285$ (13.6)

$154 / 1144(13.5)$

$163 / 1198$ (13.6)

142/1092 (13.0)

113/843 (13.4)

133/1031 (12.9)

$152 / 1065$ (14.3)

184/1304 (14.1)

$176 / 1289$ (13.7)

$152 / 1168$ (13.0)

$83 / 803$ (10.3)

$161 / 1143$ (14.1)

$141 / 1230(11.5)$

177/1278 (13.8)

120/834 (14.4)

$143 / 1055$ (13.6)

$163 / 1015$ (16.1)

150/1098 (13.7)

$172 / 1282$ (13.4)

$172 / 1232$ (14.0)

$168 / 1283$ (13.1)

$173 / 1286$ (13.5)

$183 / 1274$ (14.4)

$176 / 1300$ (13.5)

179/1267 (14.1)

$161 / 1218$ (13.2)

$121 / 1049$ (11.5)

165/1186 (13.9)

173/1134 (15.3)

$104 / 1015$ (10.2)

$125 / 1152$ (10.9)

148/1076 (13.8)

$150 / 1183$ (12.7)

180/1303 (13.8)

115/640 (18.0)

$143 / 1032$ (13.9)

128/1094 (11.7)

166/1191 (13.9)
0.001

0.046

0.043

0.191

0.257

0.030

0.447

0.037

0.489

0.249

0.057

0.006

$<0.001$

0.890

$<0.001$

0.558

0.547

0.407

$<0.001$

0.500

0.001

0.965

$<0.001$

0.003

0.021

0.002

0.357

0.008

$<0.001$

0.906

0.001

$<0.001$

$<0.001$

0.667

$<0.001$

0.263

$<0.001$

0.853

$<0.001$

0.939

ASA American Society of Anesthesiologist 
Table 2 Risk factors associated with surgical site infections in non-diabetic patients: multivariate analyses

\begin{tabular}{lll}
\hline Factors & Odds ratio & $95 \%$ confidence interval \\
\hline Persistent hyperglycemia & 1.54 & $1.03-2.31$ \\
Prolonged surgery & 1.81 & $1.23-2.65$ \\
Perioperative blood transfusion & 2.36 & $1.56-3.57$ \\
Preoperative infections & 5.04 & $3.10-8.19$ \\
Hepatectomy combined with biliary tract resection & 2.79 & $1.31-5.96$ \\
Other hepatobiliary pancreatic surgeries & 3.57 & $1.28-9.92$ \\
Small intestinal surgery & 0.54 & $0.32-0.90$ \\
Laparoscopic surgery & 0.51 & $0.26-1.00$ \\
\hline
\end{tabular}

multivariate analysis. Multivariate analysis identified persistent hyperglycemia as an independent risk factor for SSI in patients undergoing gastric surgery (OR 5.25, 95\% CI $2.25-12.25, P<0.001)$.

\section{Discussion}

It is commonly accepted that hyperglycemia affects the immune system via impaired chemotaxis, phagocytosis, overproduction of reactive oxygen species, free fatty acids, and inflammatory mediators [11]. These pathophysiologic changes also cause cellular damage and vascular dysfunction. Substantial evidence indicates that correction of hyperglycemia with insulin therapy reduces complications, including SSI, in patients undergoing cardiac and general surgery [1-5]. To determine the ability of BG control to prevent SSI, SSI rates in patients whose hyperglycemia improved were compared with those of patients with persistent hyperglycemia. In non-diabetic patients, the SSI rates increased in parallel with higher final BG categories. However, no correlation was found between hyperglycemia and SSI rates in patients with diabetes at each level of hyperglycemia. This result is in consistent with other reports $[12,13]$. In patients with diabetes, it is difficult to identify the target BG needed to prevent SSI; thus, the degree of BG control must be determined individually. Hence, in this study only the effect of hyperglycemia control in non-diabetic patients was evaluated.

Persistent hyperglycemia throughout the postoperative period (within $24 \mathrm{~h}$ ) was associated with a risk of SSI in non-diabetic patients. Early-onset transient hyperglycemia within $12 \mathrm{~h}$ (improved hyperglycemia group) did not increase the SSI rate. In the subgroup analysis, persistent hyperglycemia was an independent risk factor in patients undergoing gastric surgery. These findings demonstrate the importance of hyperglycemia control in patients with an early-onset $\mathrm{BG}$ of $\geq 150 \mathrm{mg} / \mathrm{dL}$. However, the risk of hypoglycemia should be considered in patients undergoing intensive insulin therapy with a target $\mathrm{BG}$ of $<150 \mathrm{mg} / \mathrm{dL}$ [1], especially patients admitted to the general surgical ward. Stress hyperglycemia typically resolves as surgical stress abates [6]. Approximately half of patients with an episode of early-onset hyperglycemia attained the target $\mathrm{BG}$ range within $24 \mathrm{~h}$ after surgery without insulin therapy. BG significantly decreased from 177.6 to $127.5 \mathrm{mg} / \mathrm{dL}$ without insulin use in patients with improved hyperglycemia. This raises the question of whether moderategrade hyperglycemia such as a BG of $\geq 150 \mathrm{mg} / \mathrm{dL}$ should be treated with insulin in all patients undergoing surgery. Commencement of insulin dosing after confirmation of two consecutive $\mathrm{BGs}$ of $\geq 150 \mathrm{mg} / \mathrm{dL}$ or single $\mathrm{BG}$ of $\geq 180-200 \mathrm{mg} / \mathrm{dL}[14,15]$ might be reasonable, especially in the non-surgical ICU setting. After starting insulin administration, the target BG should be set at $<150 \mathrm{mg} / \mathrm{dL}$.

This study had several limitations. First, it was a singlecenter, retrospective study. Second, there is a significant risk of bias as insulin treatment was not standardized, timing was variable, and the patient cohort/surgical interventions were heterogeneous. Third, the small number of surgical patients with diabetes in Japan might have contributed to the lack of a significant relationship between hyperglycemia and the SSI rate in patients with diabetes. Fourth, because glycemic control was dependent upon the attending physician's decisions, the performance rate of insulin treatment was low. Because of this, however, the natural course of postoperative hyperglycemia without insulin therapy was evaluated in this study. Finally, in our study the definition of stress hyperglycemia was not clear. HbA1c was not measured in patients without a history of diabetes unless they had hyperglycemia before surgery; thus, it was not possible to differentiate patients with stress hyperglycemia from those with diabetes that was not diagnosed before surgery. In conclusion, persistent hyperglycemia within $24 \mathrm{~h}$ after surgery is an independent risk factor for SSI, and remission of hyperglycemia until the morning after surgery is recommended in non-diabetic patients with postoperative hyperglycemia ( $\geq 150 \mathrm{mg} / \mathrm{dL}$ ). 


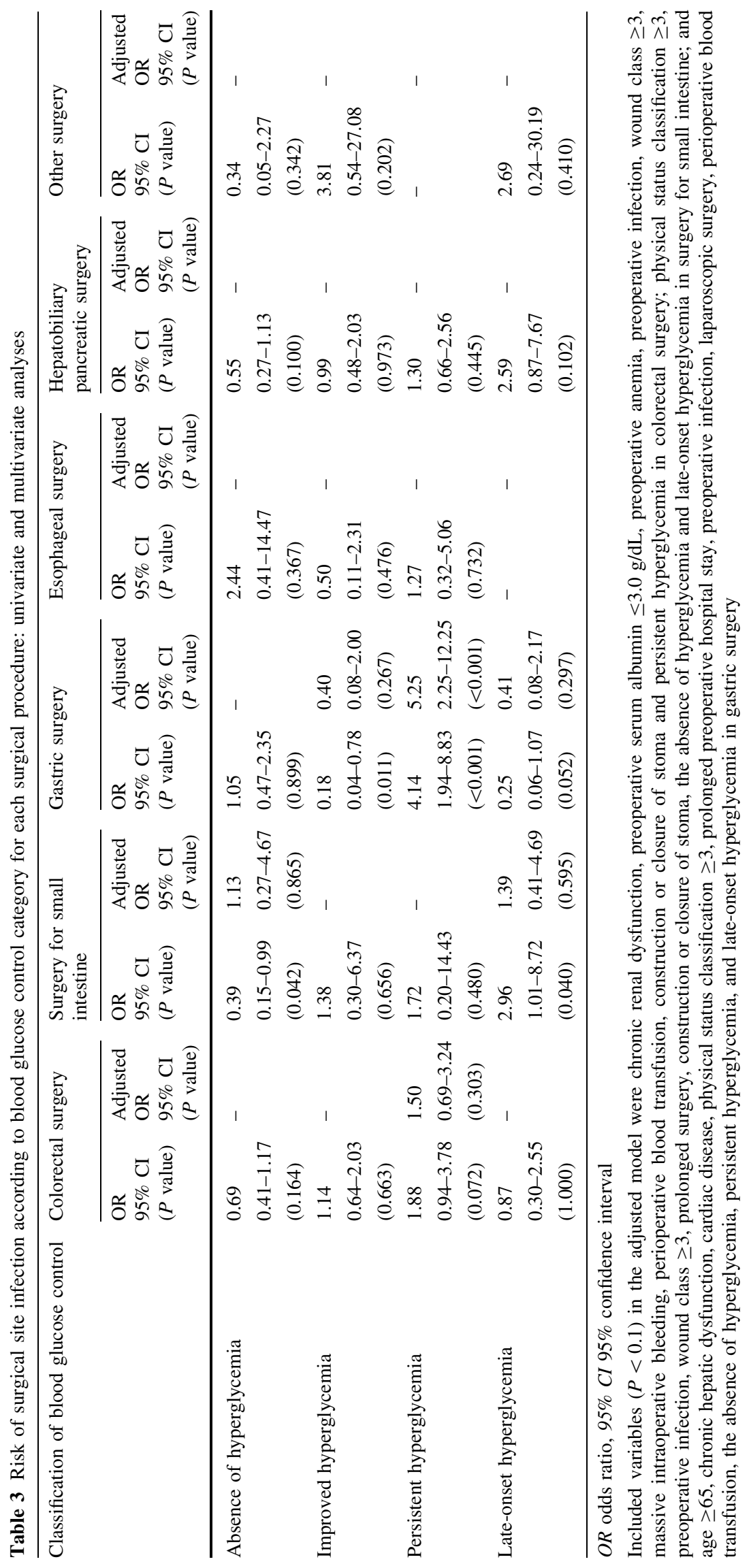


Acknowledgements No funding was received specifically for this study. We thank Angela Morben, DVM, ELS, and Dr Trish Reynolds, MBBS, FRACP, from Edanz Group for editing drafts of this manuscript.

\section{Compliance with ethical standards}

Conflict of interest All authors declare that they have no conflicts of interest.

Informed consent Because of retrospective study, informed consent was not obtained. This study was approved by the Institutional Review Board of Hyogo College of Medicine (No. 2284).

Open Access This article is licensed under a Creative Commons Attribution 4.0 International License, which permits use, sharing, adaptation, distribution and reproduction in any medium or format, as long as you give appropriate credit to the original author(s) and the source, provide a link to the Creative Commons licence, and indicate if changes were made. The images or other third party material in this article are included in the article's Creative Commons licence, unless indicated otherwise in a credit line to the material. If material is not included in the article's Creative Commons licence and your intended use is not permitted by statutory regulation or exceeds the permitted use, you will need to obtain permission directly from the copyright holder. To view a copy of this licence, visit http://creativecommons. org/licenses/by/4.0/.

\section{References}

1. de Vries FE, Gans SL, Solomkin JS et al (2016) Meta-analysis of lower perioperative blood glucose target levels for reduction of surgical-site infection. Br J Surg 104:e95-e105

2. Ata A, Lee J, Bestle SL, Desemone J et al (2010) Postoperative hyperglycemia and surgical site infection in general surgery patients. Arch Surg 145:858-864

3. Okabayashi T, Shima Y, Sumiyoshi T et al (2014) Intensive versus intermediate glucose control in surgical intensive care unit patients. Diabetes Care 37:1516-1524
4. Shanks AM, Woodrum DT, Kumar SS et al (2018) Intraoperative hyperglycemia is independently associated with infectious complications after non-cardiac surgery. BMC Anesthesiol 18:90

5. Showen A, Russell TA, Young S et al (2017) Hyperglycemia is associated with surgical site infections among general and vascular surgery patients. Am Surg 83:1108-1111

6. Duggan EW, Carlson K, Umpierrez GE (2017) Perioperative hyperglycemia management: an update. Anesthesiology 2017(126):547-560

7. Wang YY, Hu SF, Ying HM, Chen L et al (2018) Postoperative tight glycemic control significantly reduces postoperative infection rates in patients undergoing surgery: a meta-analysis. BMC Endocr Disord 18:42

8. Ban KA, Minei JP, Laronga C et al (2017) American College of Surgeons and Surgical Infection Society: surgical site infection guidelines, 2016 update. J Am Coll Surg 224:59-74

9. Peacock TS (2019) Perioperative hyperglycemia: a literature review. AORN J 109:80-86

10. CDC/NHSN surveillance definitions for surgical site infections. Centers for Disease Control and Prevention website. http://www. cdc.gov/nhsn/pdfs/pscmanual/17pscnosinfdef_current.pdf. Accessed July 10, 2019

11. Farrokhi F, Smiley D, Umpierrez GE (2011) Glycemic control in non-diabetic critically ill patients. Best Pract Res Clin Endocrinol Metab 25:813-824

12. Kotagal M, Symons RG, Hirsch IB et al (2015) Perioperative hyperglycemia and risk of adverse events among patients with and without diabetes. Ann Surg 261:97-103

13. Frisch A, Chandra P, Smiley D, Peng L et al (2010) Prevalence and clinical outcome of hyperglycemia in the perioperative period in noncardiac surgery. Diabetes Care 33:1783-1788

14. Berríos-Torres SI, Umscheid CA, Bratzler DW et al (2017) Centers for disease control and prevention guideline for the prevention of surgical site infection, 2017. JAMA Surg 152:784-791

15. Dellinger RP, Levy MM, Rhodes A et al (2013) Surviving sepsis campaign: international guidelines for management of severe sepsis and septic shock: 2012. Crit Care Med 41:580-637

Publisher's Note Springer Nature remains neutral with regard to jurisdictional claims in published maps and institutional affiliations. 